\title{
First Report of Splenic Abscesses Due to Coccidioidomycosis
}

\author{
Shabnam Assar, MD' and Tim Kuberski, MD, FIDSA² \\ ${ }^{1}$ Department of Medicine, Virginia Tech Carilion, Roanoke, Virginia USA \\ ${ }^{2}$ Department of Medicine, University of Arizona School of Medicine-Phoenix, \\ Phoenix, Arizona USA
}

\begin{abstract}
Involvement of the spleen by Coccidioides is uncommon. It is usually associated only with disseminated infection and manifests as microscopic granulomas in the spleen. We report an immunosuppressed dermatomyositis patient who presented with splenic abscesses demonstrated on a computed tomography (CT) scan which was presumed to be bacterial in origin. At splenectomy the spleen was found to be filled with aggregates of spherules due to Coccidioides. Finding large splenic abscesses on CT scan due to Coccidioides has not been previously described. We offer a hypothesis for why the abscesses occurred in this unique patient.
\end{abstract}

\section{Introduction}

Involvement of the spleen by coccidioidomycosis is usually associated with disseminated disease, however the development of splenic abscesses has not been reported. Splenic involvement by coccidioidomycosis is usually manifest as microscopic miliary splenic granulomas which have been demonstrated at autopsy in patients with disseminated infection $(1,2)$. We report an immunocompromised dermatomyositis patient who was found to have splenic abscesses due to Coccidioides spherules which were diagnosed at splenectomy.

\section{Case Presentation}

A 33-year-old Hispanic man with dermatomyositis for five years and a history of disseminated coccidioidomycosis for two years, presented to the emergency room because of left upper quadrant abdominal pain, fever and chills. Treatment of his dermatomyositis was ongoing over the previous five years and included prednisone, azathioprine and courses of intravenous immunoglobulin (IVIG) at doses of $2 \mathrm{~g} / \mathrm{kg}$ (3). Treatments of his coccidioidomycosis over the previous two years included intravenous liposomal amphotericin B followed by oral fluconazole. The patient would periodically be non-compliant about taking the fluconazole and then experience relapses of his coccidioidomycosis which required additional courses of intravenous liposomal amphotericin B.

Physical Examination and Course: Admission vital signs - temperature $38.4^{\circ} \mathrm{C}$; blood pressure 147/81 mmHg; heart rate $106 \mathrm{bpm}$; respiratory rate 18 breaths/minute and pulse oximetry $90 \%$ on room air. There was pigmentation of his face consistent with dermatomyositis, tenderness in the left upper quadrant and significant weakness of all extremities. He was bedridden and could barely move his arms and legs against 
gravity. His medications on admission were fluconazole and prednisone. An admission CT scan of the abdomen was performed because of the left upper quadrant tenderness and revealed multiple splenic abscesses (Figure 1).

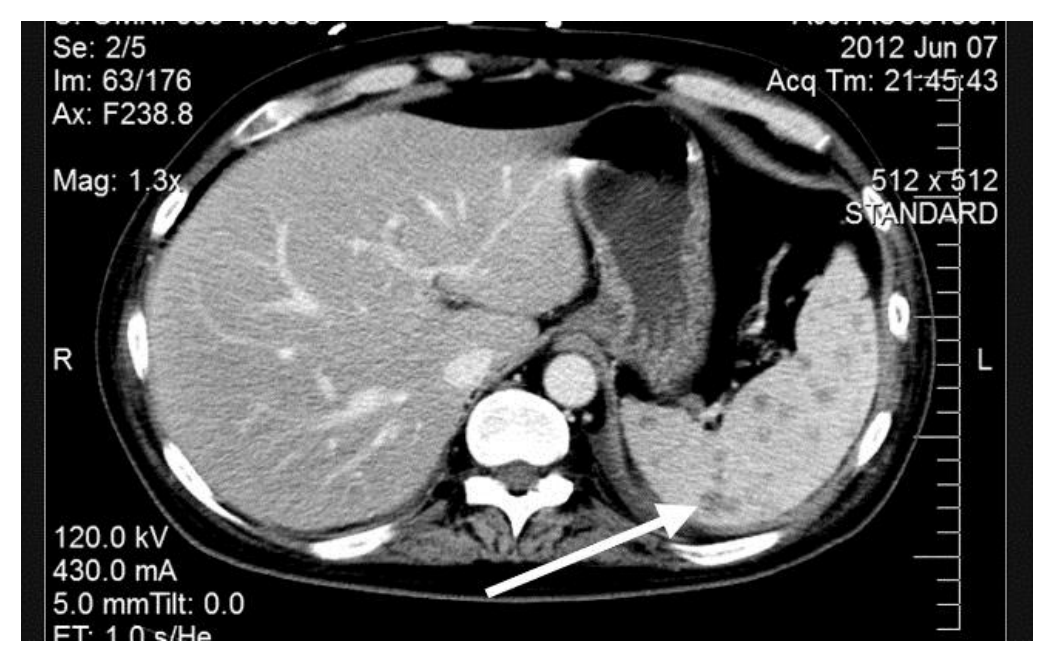

Figure 1. CT scan of abdomen demonstrating splenic abscesses (arrow).

An admission urine culture grew $>10^{5}$ colony forming Klebsiella pneumoniae which was noted on day two of hospitalization. Blood cultures were negative. It was initially believed that the splenic abscesses were due to a Klebsiella infection because of the admitting urine culture results. Prednisone was stopped on admission and the oral fluconazole continued. Piperacillin-tazobactam was started empirically on admission. In addition, IVIG was given for a presumed dermatomyositis exacerbation. On hospital day four his abdominal pain and fevers had not improved. To avoid a splenectomy, a splenic biopsy was performed to determine the cause of the splenic abnormalities. The biopsy was consistent with a Coccidioides infection. A laparoscopic splenectomy was then preformed on hospital day seven.

The pathology on the removed spleen showed multiple necrotizing granulomatous foci containing numerous aggregated Coccidioides spherules (Figure 2).

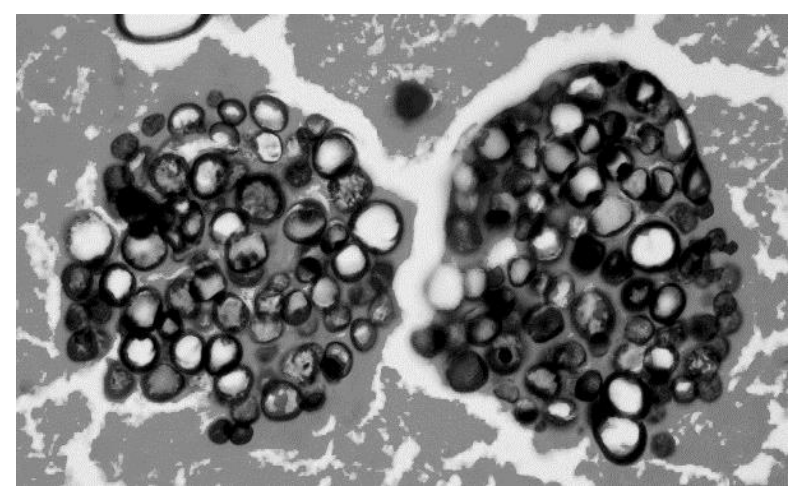

Figure 2. Pathology of splenic abscesses demonstrating aggregated Coccidioides spherules. 
Post-operatively, fluconazole was empirically replaced by voriconazole (4) and the patient was restarted on prednisone for his dermatomyositis. The fever and chills eventually resolved and he was discharged. At four months follow-up he had returned to his usual state and was encouraged to not stop taking the voriconazole.

\section{Discussion}

This patient illustrates an unusual complication of disseminated coccidioidomycosis. Prior to the advent of CT scans, splenic granulomas were described mainly at autopsy in patients with disseminated infection. Splenic involvement at autopsy was described as granulomas due to the invasion of the Coccidioides into the spleen from the blood stream. Usually there was granuloma formation described as microscopic military nodules. Reports of gross Coccidioides abscesses in the spleen have not been described.

We considered the potential reasons for the development of splenic abscesses in this unique patient. His dermatomyositis was present for about five years and the coccidioidomycosis, two years. He had received repeated doses of IVIG for flares of his dermatomyositis prior to, and after, his Coccidioides infection. Investigating his past medical history revealed that he would develop a febrile illness when off fluconazole usually due to non-compliance. The clinical presentation was consistent with either a relapse of his Coccidioides infection, an exacerbation of his dermatomyositis, or both. The febrile episodes would cause him to be admitted to the hospital, often into the intensive care unit, and then he would receive more IVIG for his dermatomyositis, as well as antifungals. It is known that fungemia occurs in immunosuppressed patients who have significant coccidioidomycosis (5). The fact that he had a large Coccidioides burden in his spleen suggests he likely experienced episodes of fungemia, presumably associated with his poor antifungal compliance.

Our hypothesis for why the abscesses formed in the spleen of this patient is illustrated in Figure 3.

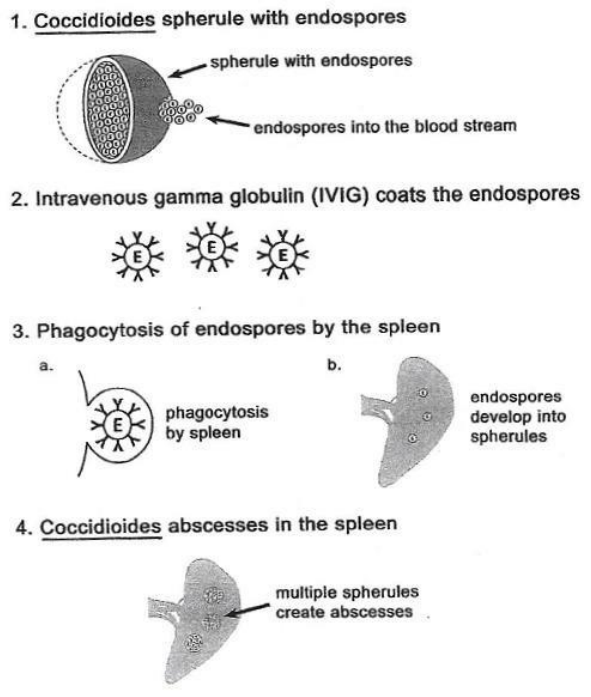

Figure 3. Hypothesis of Coccidioides abscess formation in the spleen. 
We theorized that Coccidioides endospores in the blood stream became coated with the gamma globulins when he received the IVIG given for his dermatomyositis (6). The opsonization of the organisms by the IVIG presumably facilitated the spleen to take up viable endospores into the spleen and reticuloendothelial system (Figure 3, part 3). This resulted in the localization of the organisms promoting the formation of an abscess within the spleen (Figure 3, part 4). We suggest that these unusual circumstances of fungemia and IVIG were responsible for facilitating the appearance of abscesses in this patient's spleen.

We believe true splenic abscesses are uncommon with disseminated coccidioidomycosis. The unusual circumstances of this patient's relapsing Coccidioides infection with fungemia (due to poor compliance with antifungals) and the repeated IVIG treatments for his dermatomyositis, combined to provide a reasonable explanation for why splenic abscesses occurred in this patient.

\section{References}

1. Forbus WD, Bestebreurtje AM. Coccidioidomycosis; a study of 95 cases of the disseminated type with special reference to the pathogenesis of the disease. Mil Surg. 1946 Nov;99(5):653-719. [PubMed]

2. Fiese MJ. Coccidioidomycosis: Springfield, IL: Charles C. Thomas 1958; 111.

3. Wang DX, Shu XM, Tian XL, Chen F, Zu N, Ma L, Wang GC. Intravenous immunoglobulin therapy in adult patients with polymyositis/dermatomyositis: a systematic literature review. Clin Rheumatol. 2012 May;31(5):801-6. [CrossRef] [PubMed]

4. Prabhu RM, Bonnell M, Currier BL, Orenstein R. Successful treatment of disseminated nonmeningeal coccidioidomycosis with voriconazole. Clin Infect Dis. 2004 Oct 1;39(7):e74-7. [CrossRef] [PubMed]

5. Rempe S, Sachdev MS, Bhakta R, Pineda-Roman M, Vaz A, Carlson RW. Coccidioides immitis fungemia: clinical features and survival in 33 adult patients. Heart Lung. 2007 Jan-Feb;36(1):64-71. [CrossRef] [PubMed]

6. Adkinson NF, Yunginger JW, Busse WW, et al. Middleton's Allergy Principles \& Practice (6th ed) Philadelphia, PA: Mosby, 203; 72-73. 